\title{
Evaluación de la comprensión sobre Tablas Estadísticas en estudiantes de Educación Primaria
}

\author{
Evaluation of the understanding on statistical tables in students of \\ Elementary Education
}

\author{
Alejandro Sepúlveda* \\ ORCID iD 0000-0002-5033-8400 \\ Danilo Díaz-Levicoy** \\ ORCID iD 0000-0001-8371-7899 \\ Diego Jara*** \\ ORCID iD 0000-0003-0452-9238
}

\begin{abstract}
Resumen
Este trabajo da cuenta de la organización y estructura del resultado de la respuesta observada sobre tablas estadísticas en estudiantes de Educación Primaria de escuelas municipalizadas en la provincia de Osorno-Chile. Se aplicó una prueba con cuatro preguntas abiertas a una muestra de 233 estudiantes de tercero y sexto nivel. Las respuestas fueron clasificadas según un continuo que va desde la incompetencia a la maestría: la capacidad, operaciones implicadas, consistencia y conclusiones. Los resultados muestran que las respuestas de los estudiantes se agrupan, mayoritariamente, en el enfoque de aprendizaje superficial, esto significa que, aunque entrega datos relevantes lo hace en forma aislada, los ordenan pero, falla en la conexión entre ellos. Se observó que no hay diferencias significativas entre la estructura y organización de las respuestas que elaboran los estudiantes de tercero y sexto año.
\end{abstract}

Palabras clave: Tablas estadísticas. Taxonomía SOLO. Educación Primaria.

\begin{abstract}
This work focuses on the organization and structure of the results on the response observed about statistical tables in students from a municipal elementary school from the Osorno province, in Chile. Tests with four open questions were applied to a sample of 233 students from third and sixth grades. The answers were classified according to a continuum ranging from incompetence to mastery: the ability, operations involved, consistence and conclusions. The results show that the students answers were largely grouped in a superficial learning approach, this means that, although they deliver relevant data, it does so in an isolated way, they organize the data but there is a failure in the connection between them. It was observed that there is no significant differences between the answers structure and organization from third grade or sixth grade students.
\end{abstract}

\footnotetext{
* Doctor en Ciencias de la Educación por la Universidad Academia de Humanismo Cristiano (UAHC), Chile. Académico e Investigador de la Universidad de Los Lagos (ULAGOS), Osorno, Chile. Dirección postal: Av. Fuchslocher 1305, Osorno, Chile, C.P: 5311157. E-mail: asepulve @ulagos.cl.

** Máster en Didáctica de la Matemática por la Universidad de Granada (UGR), España. Doctorando en Ciencias de la Educación de la Universidad de Granada (UGR), Granada, España. Dirección postal: Campus de Cartuja s/n, Granada, España, C.P: 18071. E-mail: dddiaz01@ hotmail.com.

**** Licenciado en Educación por la Universidad de Los Lagos (ULAGOS), Chile. Estudiante de Pedagogía en Matemática y Computación de la Universidad de Los Lagos (ULAGOS), Osorno, Chile. Dirección postal: Av. Fuschlocher 1305, Osorno, Chile, C.P: 5311157. E-mail: diegojaracar@gmail.com.
} 
Keyswords: Statistical Tables. SOLO Taxonomía. Elementary education.

\section{Introducción}

En este trabajo nos centramos en evaluar la comprensión sobre tablas estadísticas por estudiantes chilenos de tercero y sexto de Educación Primaria, estableciendo cuánto saben y si existen diferencias entre los niveles.

La investigación se ve motivada por la presencia de las tablas estadísticas, al igual que otros temas de estadística y probabilidad, que están presentes en situaciones de la vida cotidiana, como los medios de comunicación (ARTEAGA et al., 2011; BELTRÃO, 2012; EUDAVE, 2009), como por ejemplo en la sección deportiva de los periódicos en la que se muestra la ubicación de cada equipo de fútbol y su respectivo puntaje durante un torneo deportivo, o en los noticiarios para indicar las temperaturas de los próximos días en una ciudad cualquiera, entre otras situaciones de la vida cotidiana y social.

Estas representaciones son consideradas un elemento de la cultura estadística (CAZORLA; UTSUMI, 2010; GAL, 2002; WATSON, 1997), término que se refiere a la comprensión de información estadística (en forma de números, texto escrito u oral, símbolos, tabla y gráficos) con la que se interactúa en la vida diaria y que debe ser comprendida por los ciudadanos. Más aún, es una forma muy efectiva para transmitir información, en gran cantidad y en un espacio reducido, en una sociedad caracterizada por los avances tecnológicos y el flujo constante de datos e información.

Dada esta relevancia es que las directrices curriculares de Educación Primaria de diferentes países como España (MECD, 2014), Brasil (MEC, 1997), los estándares americanos (NCTM, 2000; CCSSI, 2010), en el caso de Chile, las directrices curriculares del Ministerio de Educación (MINEDUC, 2012), donde se desarrolla este estudio, observamos que en los dos primeros cursos de Educación Primaria se trabajan las tablas de conteo y las de frecuencia, aunque en los libros de texto se constata también tablas de datos y de doble entrada simples (DÍAZ-LEVICOY; MORALES; LÓPEZ-MARTÍN, 2015), además de tener una relación directa con los gráficos estadísticos por medio de las actividades de construcción y traducción (DÍAZ-LEVICOY; MORALES; LÓPEZ-MARTÍN, 2015; DÍAZ-LEVICOY; BATANERO et al., 2016), es decir, por aquellas actividades en la que construye un gráfico con la información de una tabla o cuando se construye una tabla a partir de la información de un gráfico. 


\section{Antecedentes}

\subsection{Tablas estadísticas}

Las investigaciones sobre tablas estadísticas en Educación Primaria aún son escasas y es ahí donde este trabajo se presenta como novedoso e importante.

De las investigaciones sobre tablas estadísticas podemos observar las que se relacionan con su presencia en los libros de texto y la comprensión de los estudiantes. En el primer caso observamos, por ejemplo, Amorim y Silva (2016) realizan un estudio sobre las tablas estadísticas en libros de texto de cuarto y quinto de Educación Primaria, en Brasil. Los resultados muestran que las actividades en las que se hace uso de esta representación hacen referencia a cuadros y banco de datos; que las actividades dan mayor énfasis a la lectura e interpretación, frente a la de construcción que recibe menor importancia; además, la mayoría de las actividades están relacionadas a situaciones de la vida cotidiana.

En este mismo contexto Guimarães et al. (2008) estudian las representaciones gráficas (gráficos, tablas estadísticas y tablas no estadísticas) en textos de primero a cuarto de Enseñanza Primaria. Los resultados reflejan la falta de uniformidad en la distribución de las actividades, y donde la mayoría de las que hacen referencia a las tablas no trabajan la organización de la información.

En el contexto chileno, Díaz-Levicoy, Morales y López-Martín (2015) realizan un estudio del trabajo con tablas estadísticas en los dos primeros cursos de Educación Primaria. Los resultados permiten observar la presencia de tablas de conteo, datos, frecuencia y doble entrada; el predominio de las actividades de calcular, completar y traducir; y del predominio del nivel de lectura de Curcio, de leer dentro de los datos.

También encontramos el trabajo de Estrella y Olfos (2015), quienes abordan el pensamiento transnumerativo en estudiantes de tercer año de Educación Primaria, que por medio de una actividad de ordenar y organizar los datos usan listas, seudotablas y tablas.

Espinoza (2015), en Perú, realiza una experiencia de aula con niños de 6 y 7 años, basadas en la lectura de tablas estadísticas y gráficos de barras. Los resultados indican que estos estudiantes pueden leer información directa en ambas representaciones (leer los datos). Resultados similares a los obtenidos por Ochoa (2015) en una experiencia de aula sobre el completar e interpretar tablas de doble entrada con niños de 7 y 8 años de edad, y que abordan con dificultad las de nivel 2 de leer dentro de los datos. Pero, existen trabajos como los de Pereira y Conti (2012), en donde estudiantes de tercero de Educación Primaria de Brasil son 
capaces de abordar, con éxito, actividades de lectura literal y realizan cálculos aritméticos sobre tablas de doble entrada.

Gabucio et al. (2010) aplican un cuestionario para estudiar la comprensión sobre tablas estadísticas en 205 estudiantes, desde quinto de Educación Primaria hasta segundo de Educación Secundaria, en España, donde las actividades que se desarrollan con mayor éxito son las relacionadas con leer algún dato de la tabla y conocer la estructura de la tabla.

Por lo anterior, en este estudio ofrecemos información que estimamos será novedosa, de interés y de utilidad para el desarrollo de la Educación Estadística en el mundo y, particularmente, en Chile.

\subsection{Taxonomía SOLO}

La taxonomía SOLO (Structure of the Observed Learning Outcome) (BIGGS; COLLIS, 1982) permite observar el desarrollo cognitivo de los estudiantes por medio de sus interacciones en el aula. Uno de los elementos de este marco teórico son los niveles de complejidad del conocimiento, los que permiten caracterizar la calidad de los aprendizajes. Estos niveles - preestructural, uniestructural, multiestructural, relacional y abstracto extendido - van creciendo gradualmente, donde los primeros hacen referencia a la relación y al tratamiento de la información en forma aislada y reproductiva, y en los más avanzados se hace referencia a un conocimiento profundo. Estos niveles pueden cambiar producto de la instrucción, ya que se basa en la respuesta que da el estudiante a una pregunta (BIGGS; COLLIS, 1982). En el Cuadro 1 observamos la descripción de estos niveles, de acuerdo a las capacidades y operaciones implicadas.

\begin{tabular}{|l|l|l|}
\hline \multicolumn{1}{|c|}{ Niveles } & \multicolumn{1}{|c|}{ Capacidad } & \multicolumn{1}{c|}{ Operaciones implicadas } \\
\hline $\begin{array}{l}\text { Preestructural } \\
\text { P) }\end{array}$ & $\begin{array}{l}\text { Mínima: } \\
\text { Dificultad por recordar la respuesta a la } \\
\text { cuestión planteada. } \\
\text { Respuestas confusas e indiferenciadas }\end{array}$ & $\begin{array}{l}\text { No hay interrelaciones lógicas. Tipos de } \\
\text { confusiones: negación, tautología y } \\
\text { transducción. }\end{array}$ \\
\hline $\begin{array}{l}\text { Uniestructural } \\
\text { (U) }\end{array}$ & $\begin{array}{l}\text { Baja: } \\
\text { Recuerdo de un dato relevante sobre la } \\
\text { pregunta. } \\
\text { El estudiante debe comprender la pregunta y } \\
\text { relacionarla con la respuesta al menos con } \\
\text { una operación lógica. }\end{array}$ & $\begin{array}{l}\text { Se puede generalizar, pero sólo en relación } \\
\text { a un aspecto. } \\
\text { Inducción: requiere diseñar correctamente } \\
\text { una conclusión general de una cuestión } \\
\text { particular. } \\
\text { Relacionar un aspecto particular o dato con } \\
\text { una conclusión. }\end{array}$ \\
\hline $\begin{array}{l}\text { Multiestructural } \\
\text { M) }\end{array}$ & $\begin{array}{l}\text { Media: } \\
\text { Recuerdo de dos o más datos relevantes, } \\
\text { aunque de forma aislada. }\end{array}$ & $\begin{array}{l}\text { Se puede generalizar en relación a algunos } \\
\text { aspectos limitados e independientes. } \\
\text { Ordena adecuadamente varios datos } \\
\text { relevantes, pero falla la conexión entre } \\
\text { ellos. } \\
\text { Generalmente contiene: y... también.... }\end{array}$ \\
\hline Relacional ( R) & Alta: & Inducción: Se puede generalizar dentro de \\
\hline
\end{tabular}




\begin{tabular}{|l|l|l|}
\hline Niveles & \multicolumn{1}{|c|}{ Capacidad } & \multicolumn{1}{c|}{ Operaciones implicadas } \\
\hline & $\begin{array}{l}\text { Se interrelacionan los datos o conceptos } \\
\text { relevantes. }\end{array}$ & $\begin{array}{l}\text { un contexto o experiencia dada, utilizando } \\
\text { los aspectos (contenidos) implicados en la } \\
\text { situación. } \\
\text { Da un conjunto de conceptos o principios } \\
\text { relacionados. }\end{array}$ \\
\hline $\begin{array}{l}\text { Abstracción } \\
\text { Extendida (AE) }\end{array}$ & $\begin{array}{l}\text { Máxima: } \\
\text { Datos relevantes interrelacionados. } \\
\text { El estudiante necesita no únicamente } \\
\text { relacionar la información dada, sino } \\
\text { también comprender la relevancia de los } \\
\text { principios abstractos primordiales de los } \\
\text { cuales puede deducir una hipótesis y } \\
\text { aplicarla a una situación que no ha sido } \\
\text { dada. }\end{array}$ & $\begin{array}{l}\text { Deducción e inducción. Se puede } \\
\text { generalizar a situaciones no experimentales. } \\
\text { Hay varias características de este nivel que } \\
\text { debemos tener en cuenta: } \\
\text { a) la introducción de un principio abstracto } \\
\text { que no se ha dado directamente en los datos. } \\
\text { b) La deducción desde este principio y la } \\
\text { comprobación de esta deducción } \\
\text { confrontándola con los datos. } \\
\text { c) La introducción de una analogía que sea } \\
\text { compatible con estos principios, pero no se } \\
\text { haya dado con los datos. }\end{array}$ \\
& $\begin{array}{l}\text { d) El resultado puede ser indeterminado (los } \\
\text { eventos pueden haber sido diferentes en } \\
\text { distintas circunstancias). }\end{array}$ \\
\hline
\end{tabular}

Cuadro 1 - Descripción de los niveles de la taxonomía SOLO

Fuente: Biggs y Collis (1982).

\section{Formulación del problema y objetivos}

La comprensión es la habilidad de pensar y actuar con flexibilidad a partir de lo que uno sabe, en otras palabras, la capacidad de desempeño flexible (STONE, 1999). La comprensión del aprendizaje es y ha sido una preocupación de larga data. Según los datos del Consejo Nacional de la Cultura y las Artes (CNCA, 2011) la mitad de los estudiantes no comprende lo que lee y, un $40 \%$ de los estudiantes de Educación Primaria y Secundaria, de buen rendimiento escolar, no comprenden la materia (SEPÚLVEDA; DELGADO, 2007).

En relación a lo anterior, en este estudio pretende determinar cuál es la estructura del resultado del aprendizaje, sobre tablas estadísticas, en estudiantes de Educación Primaria de escuelas municipalizadas, desde la taxonomía SOLO.

\section{Marco metodológico}

\subsection{Metodología y descripción del instrumento}

En este estudio se evalúan los resultados del aprendizaje observados en estudiantes de tercer y sexto año de Educación Primaria de escuelas municipalizadas en relación a las tablas estadísticas según la taxonomía SOLO.

Para ello se siguió una metodología cuantitativa no experimental, de tipo descriptiva y 
de corte de datos transversal, esto es, tomados en un solo momento (HERNÁNDEZ; FERNÁNDEZ; BAPTISTA, 2010). Se pesquisa información a través de la adaptación del cuestionario diseñado por Díaz-Levicoy, Morales, et al. (2016) de acuerdo a los resultados de un estudio de estas representaciones en libros de texto en los dos primeros años de Educación Primaria en Chile (DÍAZ-LEVICOY; MORALES; LÓPEZ-MARTÍN, 2015). Las actividades del cuestionario siguen una estructura de superítem, de acuerdo a los niveles de la taxonomía $S O L O$, es decir, consta de cuatro preguntas y si se responde correctamente la primera de ellas supone que el estudiante es capaz de alcanzar, al menos, el nivel uniestructural; si responde correctamente la segunda pregunta supone que alcanza, al menos, el nivel multiestructural; y, del mismo modo, alcanzaría los niveles relacional y de abstracción extendida, cuando responda correctamente a la tercera y cuarta preguntas, respectivamente.

\subsection{Validación del instrumento}

Tras la adaptación del instrumento, fue necesario evaluar su pertinencia con el objetivo propuesto. Por lo que se recurrió al criterio de tres expertos (uno competente en la taxonomía SOLO, uno en educación y otro en Educación Matemática), que permitieron verificar la coherencia del cuestionario con la estructura de la taxonomía SOLO. Luego de esto, se aplicó a una muestra reducida de quince estudiantes de Educación Primaria, para verificar la complejidad de las preguntas de acuerdo a los niveles SOLO y el tiempo que ocupa su resolución.

En tercer lugar, se realizó una validación interjueces (BIGGS; COLLIS, 1982), es decir, se crearon dos grupos constituidos por tres jueces cada uno, que no han participado en la validación anterior, los que tenían la tarea de analizar las respuestas de los primeros tres expertos. Cada grupo de jueces debía situar cada respuesta según los niveles SOLO, y estos se contrastaron con los obtenidos por cada grupo, para discutir en caso de disparidad de criterios. Luego de estos tres procesos se obtiene la versión definitiva del cuestionario (ver Anexo 1).

\subsection{Muestra y aplicación del instrumento}

La muestra que ha sido parte de este estudio estuvo conformada por 233 estudiantes de tercero y sexto año de Educación Primaria, según la distribución que observamos en la Tabla 1. 
Tabla 1 - Muestra de estudiantes de Educación Primaria

\begin{tabular}{cc}
\hline Nivel educativo & Muestra \\
\hline Tercer año & 147 \\
Sexto año & 86 \\
\hline Total & 233 \\
\hline
\end{tabular}

Fuente: elaborado por los autores.

La aplicación del cuestionario se realizó durante el segundo trimestre del año 2016, tras convenir el ingreso a las salas de clases, en un determinado horario, donde asistieron los investigadores junto al profesor del curso. Dicha aplicación consideraba, como máximo, 45 minutos. La recolección de los datos se llevó a cabo aproximadamente en un mes.

\subsection{Codificación y asignación de niveles SOLO a las respuestas}

Para la codificación de los datos, a la respuesta de los estudiantes a cada superítem se le asocia un vector de 4 componentes, cualitativos y ordenados. Por ejemplo, para el estudiante $E$ la evaluación correspondiente al superítem 2 está constituida por el vector (U, M, $\mathrm{R}, \mathrm{nA})$ que indica que este alumno ha respondido correctamente hasta el nivel relacional, ya que en el nivel abstracto se le asigna el vector nA que significa que no responde correctamente esta pregunta.

Para asignar un único nivel SOLO a cada estudiante, se analizan los cuatro vectores descritos en el Cuadro 2. Para ello se usa el criterio, arbitrario, cuyo nivel de exigencia, en este caso, permite a lo sumo un fallo y, por lo tanto, responder al menos tres ítems correctamente (75\% de logro), siguiendo criterios de investigaciones previas (SEPÚLVEDA, 2004). Es así como al estudiante $E$ se le asigna el nivel Multiestructural, pues en respuestas de nivel relacional y abstracto no responde correctamente los tres ítems exigidos como mínimo.

\begin{tabular}{|c|c|c|c|c|}
\hline Superítems & Ítem 1 & Ítem 2 & Ítem 3 & Ítem 4 \\
\hline Superítem 1 & $\mathrm{U}$ & $\mathrm{M}$ & $\mathrm{R}$ & $\mathrm{A}$ \\
\hline Superítem 2 & $\mathrm{U}$ & $\mathrm{M}$ & $\mathrm{R}$ & $\mathrm{nA}$ \\
\hline Superítem 3 & $\mathrm{U}$ & $\mathrm{M}$ & $\mathrm{nR}$ & $\mathrm{nA}$ \\
\hline Superítem 4 & $\mathrm{U}$ & $\mathrm{M}$ & $\mathrm{nR}$ & $\mathrm{nA}$ \\
\hline \multicolumn{4}{|l}{} \\
\hline
\end{tabular}

Cuadro 2 - Ejemplo del resultado de la evaluación SOLO de la estudiante $E$ Fuente: elaborado por los autores.

\section{Resultados}

A continuación, se exponen los principales resultados obtenidos en la aplicación del instrumento a los estudiantes de Educación Primaria, los que se estructuran de la siguiente forma: resultados a niveles generales, rendimiento en matemática, según nivel que cursan los 
estudiantes y la estructura y organización de las respuestas; realizando comparaciones, cuando sea posible, entre los estudiantes de tercero y sexto.

\subsection{Respuestas de la muestra según niveles SOLO}

La Figura 1 muestra la clasificación de las respuestas de los estudiantes según niveles de la taxonomía SOLO. Se observa que el $62,7 \%$ se clasifica en un nivel uniestructural (capacidad baja). El 21\% de las respuestas califican en el nivel multiestructural (capacidad media) y 14,9\% en el nivel preestructural (no demuestra capacidad). Solo el 1,2\% alcanza el nivel relacional (Capacidad alta) y no existen respuestas de estudiantes que califiquen en el nivel de abstracción extendida, correspondiente a la capacidad máxima en la cual los estudiantes son capaces de deducir e inducir, pudiendo generalizar y aplicar en nuevos contextos. Ejemplos de lo dicho anteriormente son:

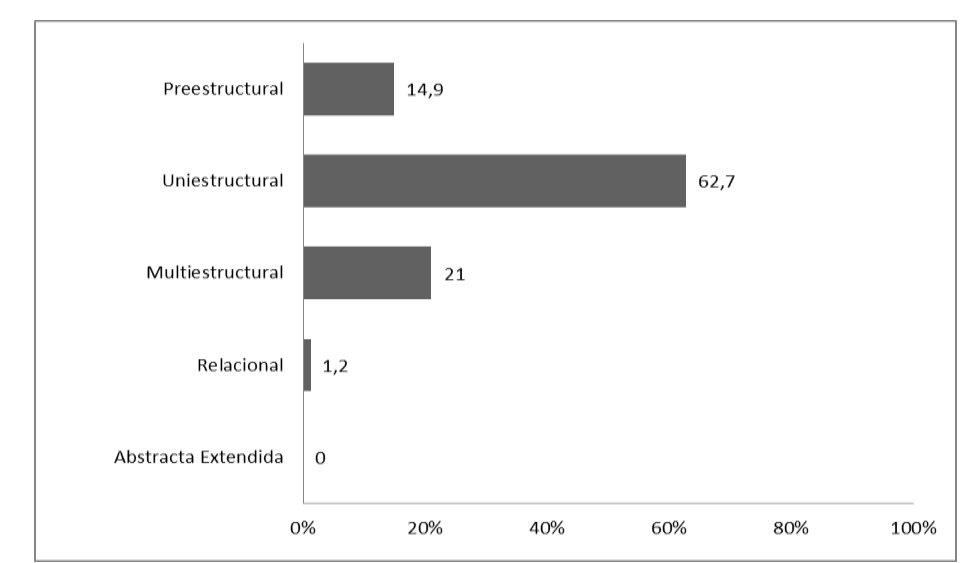

Figura 1 - Respuestas de estudiantes de la muestra según niveles SOLO Fuente: elaborado por los autores.

Frente a la pregunta ¿Invente un problema que se puede resolver con la información presente en la Tabla? del Ítem 3 (Anexo 1), un ejemplo de respuesta evaluada como de tipo uniestructural es la del estudiante 43, ya que se limita a repetir una pregunta dada en el ítem, tiene una dificultad baja, porque es muy concreta y demanda poca elaboración.

¿Cuántos estudiantes prefieren el durazno?

(Estudiante 43, 2016).

En relación a preguntas de tipo abstracción extendida, por ejemplo, a ¿Qué actividades podría hacer la directora para aumentar su aprobación? en el ítem 4, una de las respuestas no califica como correcta es la mencionada por el estúdiate 38 , ya evidencia un razonamiento bajo, es decir, no se ha realizado un análisis profundo de la situación.

Organizar mejor su trabajo.

(Estudiante 38, 2016). 


\subsection{Respuestas por categorías SOLO según nivel educativo}

En la Figura 2 se muestran resultados comparativos de la clasificación de las respuestas de los estudiantes de tercero y sexto curso. El nivel uniestructural (capacidad baja) agrupa un $78,5 \%$ de las respuestas de los estudiantes de tercero y 46,9\% en sexto de Educación Primaria. En el nivel multiestructural (capacidad media) los de sexto de primaria alcanzan un $34,9 \%$ sobre el $7,1 \%$ de tercero. La ausencia de capacidad, que corresponde al nivel preestructural alcanza el 15,6\% en sexto básico y 14,2\% de tercero. Respecto al dominio de la capacidad alta (nivel relacional), en sexto de primaria llegan al 2,4\%, sin que existan respuestas de representantes de tercero de Educación Primaria en este nivel. Finalmente, no existen respuestas de estudiantes que hayan alcanzado el nivel de abstracción extendida (capacidad máxima).

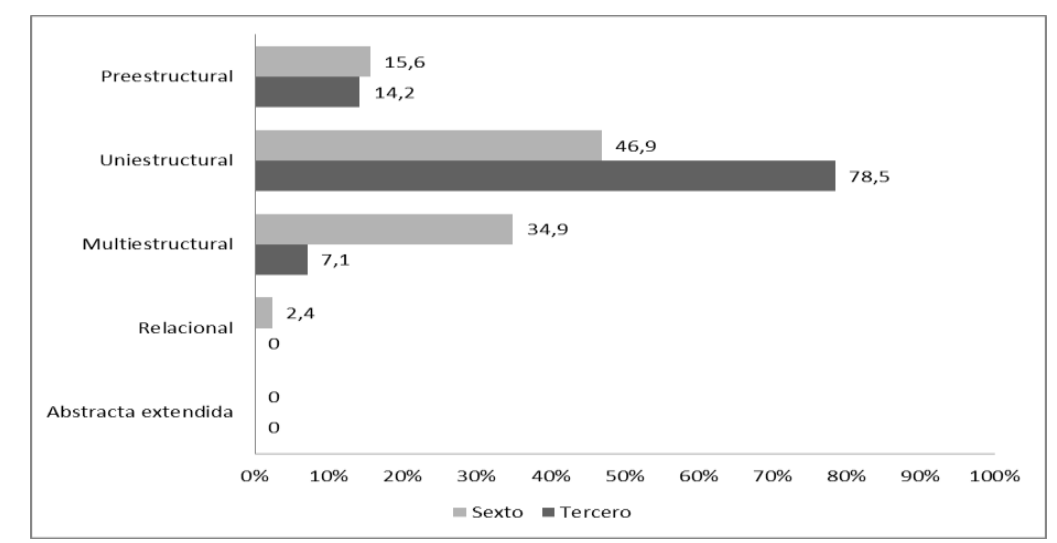

Figura 2 - Respuestas de estudiantes de tercero y sexto de primaria según los niveles SOLO Fuente: elaborado por los autores.

\subsection{Respuestas de los estudiantes según profundidad del conocimiento}

Para calificar las respuestas de los estudiantes, los autores de este estudio, se apoyan en la categoría propuesta por Marton, Hounsell y Entwistle (1984) quienes homologan el conocimiento profundo a los niveles SOLO: abstracto extendido y relacional y, al conocimiento superficial a los niveles SOLO: preestructural, uniestructural y multiestructural.

Con esta organización, la conexión entre el aprendizaje desde la estructura de la respuesta otorgada por los estudiantes y el enfoque de aprendizaje profundo y superficial, se pudo constatar que, mayoritariamente, las respuestas se agrupan en el enfoque de aprendizaje superficial $(98,8 \%)$, como se puede observar de la Tabla 2, que corresponde a los niveles inferiores de la taxonomía $S O L O$, y que se circunscriben al tratamiento de la información de 
manera aislada y reproductiva.

Tabla 2 - Porcentaje de respuesta de los estudiantes según nivel de conocimiento

\begin{tabular}{cccc}
\hline \multicolumn{2}{c}{ Conocimiento superficial } & \multicolumn{2}{c}{ Conocimiento profundo } \\
\hline Preestructural & Uniestructural & Multiestructural & Relacional \\
& 98,8 & & Abstracta extendida \\
\hline
\end{tabular}

Fuente: elaborado por los autores.

Por ejemplo, la respuesta que mostramos a continuación se limita a redactar una pregunta extraída de la tabla de manera literal, sin considerar otras situaciones.

¿Cuántos estudiantes prefieren la naranja?

(Estudiante 17, 2016).

De la misma forma, se muestra que el 1,2\% de las respuestas de los estudiantes califica en un conocimiento profundo. Es decir, respuestas que pueden relacionar el tema abordado y extrapolarlo a otras situaciones, teniendo una visión global de la información. Así como se muestra en la siguiente respuesta:

Un conjunto de actividades como mejorar la comunicación con los profesores, dar más participación y que la directora sea un ejemplo a seguir.

(Estudiante 214, 2016).

\subsection{Respuestas de los estudiantes según rendimiento en matemática y niveles SOLO}

La Figura 3 muestra la puntuación y clasificación de las respuestas de los estudiantes de tercero de Educación Primaria según niveles SOLO, de acuerdo a una escala de calificación por rendimiento escolar construida por los autores, a la luz de la escala de rendimiento escolar Chilena.

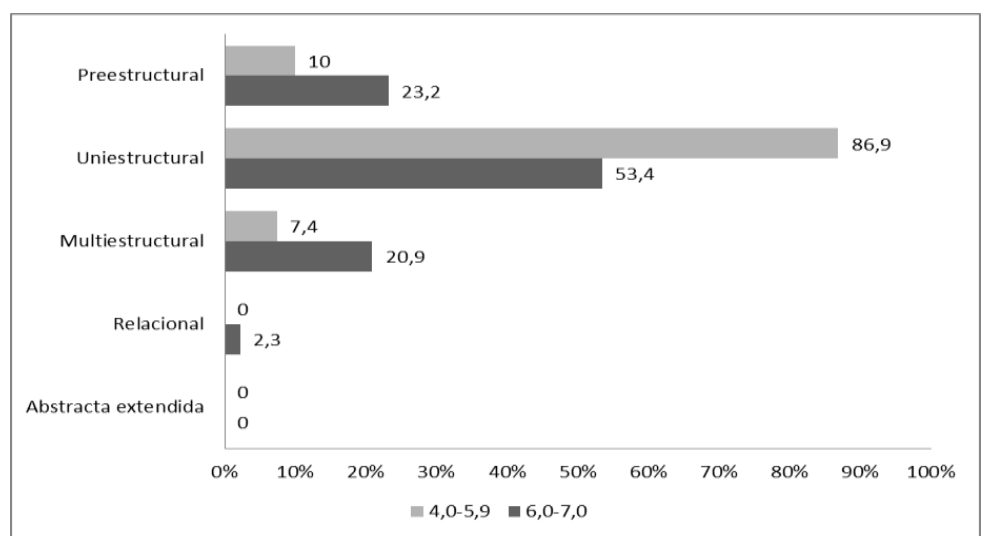

Figura 3 - Respuestas de estudiantes de tercer curso según rendimiento en matemática Fuente: elaborado por los autores.

En Chile, la Escala de Rendimiento Escolar define el rango de notas entre 4,0 y 5,9 como suficiente a buena, y entre el intervalo 6,0 y 7,0 muy buen rendimiento. Este criterio permite corroborar si el rendimiento escolar guarda una relación de proporción directa respecto de los niveles de la taxonomía SOLO. Si bien, respuestas de estudiantes con mejor 
rendimiento escolar (6,0-7,0) ocupan mayor porcentaje en el nivel multiestructural, 20,9\% respecto del 7,4\% con rendimiento entre 4,0-5,9 y alcanzan en un 2,3\% el nivel relacional, se contradice con los porcentajes del nivel preestructural en el cual estudiantes de mejor rendimiento $(6,0-7,0)$, superan en una proporción mayor al doble a estudiantes de menor rendimiento $(4,0-5,9)$, es decir, un $23,2 \%$ contra $10 \%$, respectivamente. Los logros en el nivel uniestructural, demuestran una alta concentración de respuestas de estudiantes de rendimiento menor $(4,0-5,9), 86,9 \%$ respecto del $53,4 \%$ de quienes se encuentran en el tramo de rendimiento superior.

Ante la consulta a los estudiantes, ¿Si se hace la misma pregunta a todos los estudiantes de la escuela los resultados serán similares?. Los estudiantes de rendimiento escolar entre 6,0 y 7,0 formularon respuestas como:

No porque hay compañeros flojos y aplicados y según eso es su respuesta.

(Estudiante 232, 2016).

En cambio, los estudiantes de rendimiento entre 4,0 a 5,9, redactaron respuestas como la siguiente:

Estoy de acuerdo.

(Estudiante 113, 2016).

Del mismo modo, la Figura 4 da cuenta que, según el criterio rendimiento escolar, las respuestas de estudiantes de sexto de Educación Primaria, a la luz de la taxonomía SOLO. En el nivel preestructural los estudiantes de rendimiento escolar entre 6,0 y 7, 0 agrupa el 5\% de sus respuestas, contra el 29,1\% de estudiantes de rendimiento entre 4,0-5,9. En el nivel uniestructural se observa que el $42,5 \%$ de las respuestas son de estudiantes de buen rendimiento escolar en matemática, contra 33,3\% de estudiantes de rendimiento entre 4,0 y 5,9. En el nivel relacional y abstracto extendido solo están presentes estudiantes de rendimiento escolar entre 6,0 y 7,0 con un $7,5 \%$ y $5 \%$ respectivamente.

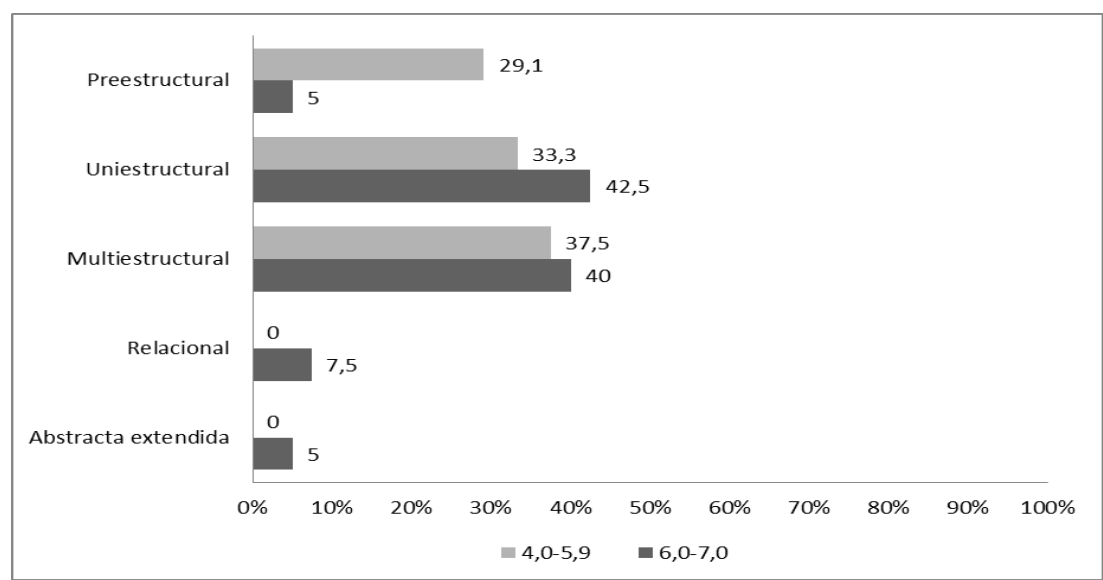

Figura 4 - Respuestas de estudiantes de sexto curso según rendimiento en matemática Fuente: elaborado por los autores. 


\subsection{Respuestas de los estudiantes de rendimiento escolar entre 4,0 y 5,9}

En la Figura 5 se muestra que al comparar las respuestas de los estudiantes con rendimiento entre 4,0 y 5,9 de ambos niveles en la taxonomía SOLO, no hay estudiantes que superen el nivel multiestructural. Sobre esta distribución, los cursos de tercero se concentran en un $86,9 \%$ el nivel uniestructural, seguido por un $10 \%$ en el nivel preestructural y $7,4 \%$ del multiestructural, mientras que los cursos de sexto ocupan en mayor proporción el nivel multiestructural en un $37,5 \%$, seguido por el 33,3\% en el nivel uniestructural y el $29,1 \%$ en el nivel preestructural.

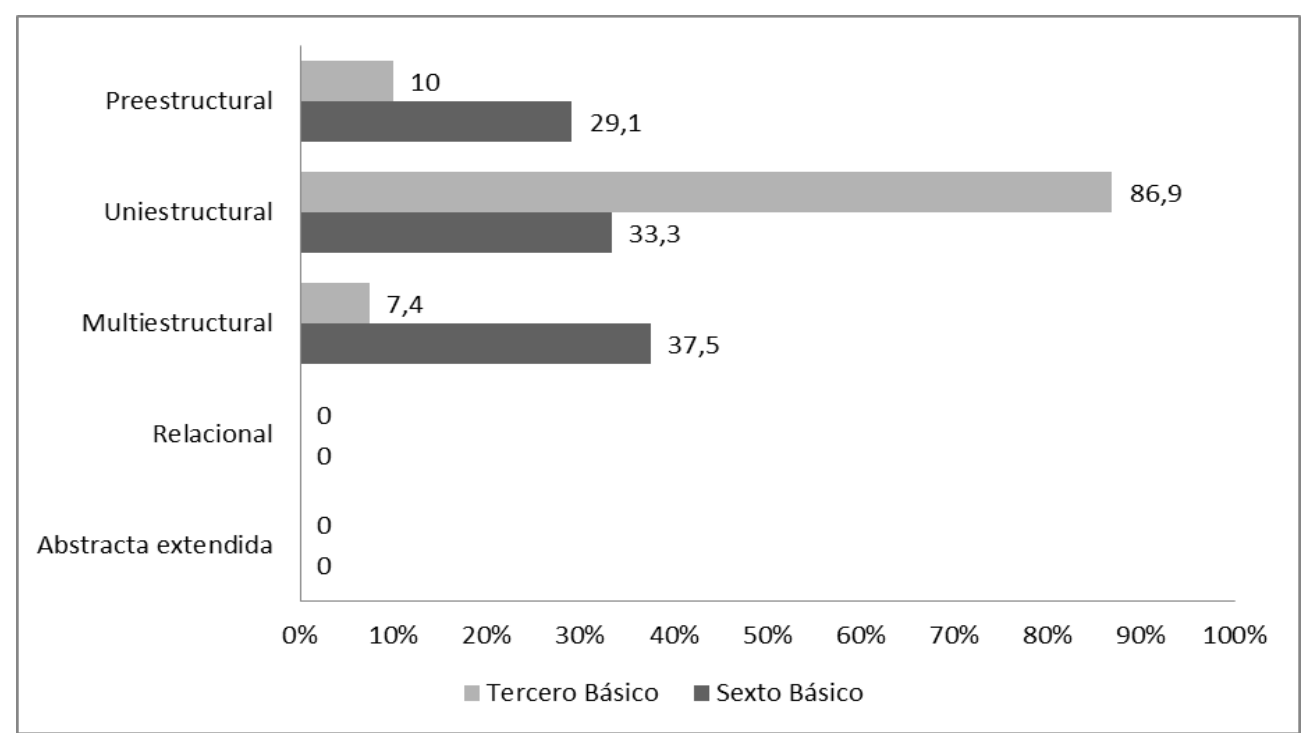

Figura 5 - Respuestas de estudiantes de tercero y sexto curso con rendimiento en matemática entre [4, 6[ Fuente: elaborado por los autores.

\subsection{Respuestas de los estudiantes de rendimiento entre 6,0-7,0}

En la Figura 6 se observa que las respuestas de los estudiantes de sexto se distribuyen en todos los niveles de la taxonomía SOLO, logrando un 5\% en nivel de abstracción extendida y un 7,5\% en el nivel relacional. En este último, también un 2,3\% de los estudiantes de tercero se encuentran presentes, no así en el nivel de capacidad máxima. La mayor concentración de respuestas se ubica en el nivel uniestructural, siendo más de la mitad de tercer año $(53,4 \%)$ y el $42,5 \%$ de sexto. Luego, un segundo grupo mayoritario se concentra en el nivel multiestructural, prevaleciendo el sexto año con el $40 \%$ sobre el $20,9 \%$ de los estudiantes de tercero. Finalmente, el 23,2\% de respuestas de estudiantes de tercero se encuentran en el nivel preestructural, respecto del $5 \%$ que corresponde a estudiantes de sexto. 


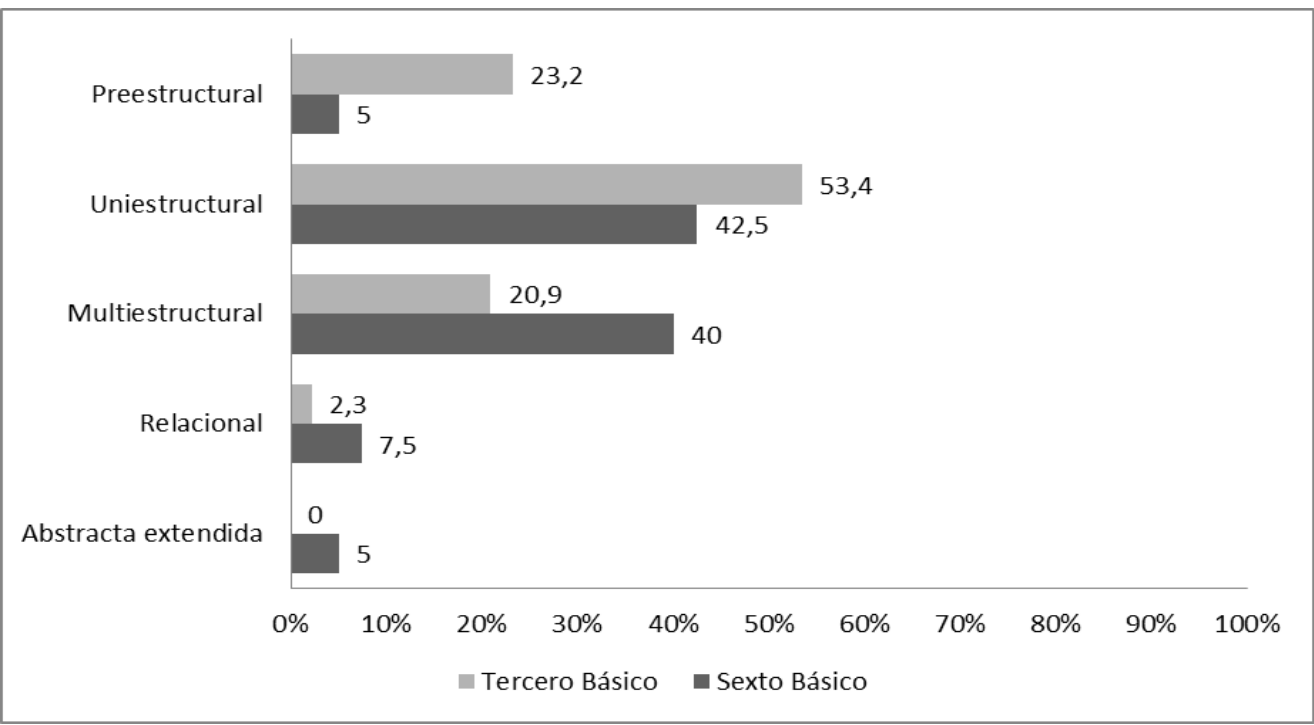

Figura 6 - Respuestas de estudiantes de tercero y sexto curso con rendimiento en matemática entre [6, 7] Fuente: elaborado por los autores.

\subsection{Análisis estadístico de los datos}

\subsubsection{Diferencias entre las respuestas de los estudiantes según rendimiento escolar}

En este apartado se exponen los resultados del análisis estadístico realizado a los datos. Las preguntas orientadoras fueron: ¿Difieren las notas en matemática entre estudiantes de $3^{\circ}$ y $6^{\circ}$ de Educación Primaria?, ¿Existe asociación de las notas por intervalos 4,0 a 5,9 y 6,0 a 7,0 entre estudiantes de $3^{\circ}$ y $6^{\circ}$ de Educación Primaria?

En la primera interrogante se pretende dar respuesta a: ¿El rendimiento escolar en matemática depende del nivel?

Si el rendimiento escolar fuese igual en ambos niveles, podríamos afirmar que el rendimiento no depende del nivel. Por lo tanto, estudiar la relación entre el rendimiento escolar y el nivel equivale a realizar un contraste de comparación de medidas de tendencia central.

En efecto, para evaluar si las notas difieren entre estudiantes de $3^{\circ}$ y $6^{\circ}$ curso usamos el test estadístico T-Student para muestras independientes. Sin embargo, al no cumplirse el supuesto de normalidad (Mediante el test Kolgomorov-Smirnov) en cada uno de los grupos que genera la variable curso, se optó por su variante no paramétrica denominada U de MannWhitney.

El contraste no paramétrico detectó que no existen diferencias significativas $(\mathrm{U}=5839,0$; valor- $\mathrm{p}=0,33>0,05)$ entre las medianas de notas obtenidas entre estudiantes de $3^{\circ}$ y $6^{\circ}$ básico en la prueba de matemática. En consecuencia, no hay asociación entre las 
calificaciones obtenidas por los estudiantes y el curso de donde provienen.

Respecto a la segunda interrogante, se pretende responder ¿Existe asociación de notas por intervalos entre estudiantes de $3^{\circ}$ y $6^{\circ}$ de Educación Primaria?

La Tabla 3 muestra la distribución porcentual de estudiantes por nivel educativo y rendimiento escolar en matemática. Aquí, se puede observar la cantidad de estudiantes y frecuencia (expresada en \%) respecto al valor total dentro de cada grupo $\left(3^{\circ}=147\right.$ estudiantes y $6^{\circ}=86$ estudiantes)

Tabla 3 - Calificación de los estudiantes

\begin{tabular}{cccc}
\hline Rango de calificación & Tercero $(n=147)$ & Sexto $(n=86)$ & Total $(n=233)$ \\
\hline 40 a 59 & $47,6 \%$ & $53,5 \%$ & $49,8 \%$ \\
60 a 70 & $52,4 \%$ & $46,5 \%$ & $50,2 \%$ \\
\hline
\end{tabular}

Fuente: elaborado por los autores.

Cada valor representa la proporción de veces que aparece cada valor en la muestra de cada curso. Por ejemplo, se puede afirmar que, el 46,5\% del total de estudiantes de $6^{\circ}$ básico tienen notas comprendidas entre 6,0 y 7,0.

Si las variables nivel y rango de notas fuesen independientes, las notas serían similares, que es tanto como afirmar que cualquiera sea el curso $\left(3^{\circ}\right.$ ó $\left.6^{\circ}\right)$, la distribución de notas es la misma.

En la medida en que la distribución anterior existan diferencias, se estará alejando del concepto de independencia y habrá que admitir que las variables están asociadas.

Para responder con precisión y evidenciar la existencia o no de asociación entre las variables nivel y rango de notas, se realizó un contraste de hipótesis mediante la prueba Chicuadrado de Pearson, admitiendo un nivel de significancia igual a 0,05.

El contraste detectó que no existen diferencias significativas (Chi cuadrado=0,748; g.l $=1$ y valor- $\mathrm{p}=0,387>0,05)$ entre las variables nivel y rango de notas. En consecuencia, las variables rango de notas y nivel son independientes, por tanto, no hay asociación entre las mismas.

La prueba anterior nos indica que la distribución de calificaciones o notas es similar no importando el curso de donde provienen.

\section{Discusión y Conclusión}

En las últimas décadas, los resultados de las investigaciones han demostrado que los estudiantes no recuerdan ni comprenden gran parte de lo que se les enseña (HIRSCH, 2007).

En este sentido, el estudio pretendió determinar cuál es la estructura del resultado del 
aprendizaje de los estudiantes sobre tablas estadísticas a la luz de la taxonomía SOLO. Sobre este particular, se pudo constatar que los estudiantes de Educación Primaria de escuelas municipalizadas entregan, en sus respuestas, dos o más datos relevantes, aunque de forma aislada, generalizan en relación a algunos aspectos, ordenan adecuadamente varios datos importantes pero, falla la conexión entre ellos, sus conclusiones no están interrelacionadas y la explicación se centra en un aspecto aislado del dato. Esto significa, de acuerdo a la taxonomía SOLO, que las respuestas de los estudiantes de enseñanza primaria se agrupan en el nivel de aprendizaje definido como multiestructural.

Las respuestas en la temática tablas estadísticas, elaboradas por los estudiantes, sean estos de tercero o sexto año, se agrupan, mayoritariamente, en el enfoque de aprendizaje superficial, esto significa que son reproductivas, aisladas, irrelevantes. Como consecuencia, prácticamente no se observaron respuestas en la categoría superior de la taxonomía que corresponde a comprensión del aprendizaje, hallazgos que se corresponden con lo encontrado por Sepúlveda (2004), en el que se da cuenta que la mayoría de las respuestas de los estudiantes, se distribuyen en las categorías inferiores de la taxonomía.

Se observa que no hay diferencias significativas entre la estructura y organización de las respuestas, que elaboran los estudiantes de tercer y sexto año. En consecuencia, en este caso, la comprensión del aprendizaje no dependió del nivel educativo que cursa el estudiante de primaria.

El contraste no paramétrico detectó que no existen diferencias significativas entre las medianas de notas obtenidas entre estudiantes de $3^{\circ}$ y $6^{\circ}$. En consecuencia, no hay asociación entre las calificaciones obtenidas por los estudiantes y el nivel de estudio que cursan.

\section{Referencias}

AMORIM, N. D.; SILVA, R. L. Apresentação e utilização de tabelas em livros didáticos de matemática do $4^{\circ}$ e $5^{\circ}$ anos do ensino fundamental. EM TEIA - Revista de Educação Matemática e Tecnológica Iberoamericana, Recife, v. 7, n. 1, p. 1-21, 2016.

ARTEAGA, P. et al. Las tablas y gráficos estadísticos como objetos culturales. Números, La Laguna, v. 76, p. 55-67, 2011.

BELTRÃO, T. M. S. Uma análise da transposição didática externa com base no que propõem documentos oficiais para o ensino de gráficos estatísticos. Revista Paranaense de Educação Matemática, Campo Mourão, v. 1, n.1, p. 131-152, 2012.

BIGGS, J.; COLLIS, K. Evaluating the quality of learning: the taxonomy. New York: Academic Press, 1982.

CAZORLA, I.; UTSUMI, M. C. Reflexões sobre o ensino de estatística na educação básica. In: CAZORLA, I.; SANTANA, E. (Ed.). Do tratamento da informação ao letramento estatístico. 
Itabuna: Via Litterarum, 2010. p. 9-18.

COMMON CORE STATE STANDARDS INITIATIVE - CCSSI. Common Core State Standards for Mathematics. Washington: National Governors Association for Best Practices and the Council of Chief State School Officers, 2010.

CONSEJO NACIONAL DE LA CULTURA Y LAS ARTES - CNCA. Plan Nacional de Fomento de la Lectura. Lee Chile Lee. Santiago: Consejo Nacional de la Cultura y las Artes, 2011

DÍAZ-LEVICOY, D.; BATANERO, C. et al. Gráficos estadísticos en libros de texto de Educación Primaria: un estudio comparativo entre España y Chile. BOLEMA - Boletim de Educação Matemática, Rio Claro, v. 30, n. 55, p. 713-737, ago. 2016.

DÍAZ-LEVICOY, D.; MORALES, R. et al. Validación de un cuestionario para evaluar la comprensión sobre tablas estadísticas en Educación Primaria. In: CONGRESO INTERNACIONAL DE INVESTIGACIÓN E INNOVACIÓN EN EDUCACIÓN INFANTIL Y PRIMARIA, 4., 2016, Murcia. Actas... Murcia: Universidad de Murcia, 2016. p. 518-524.

DÍAZ-LEVICOY, D.; MORALES, R.; LÓPEZ-MARTÍN, M. M. Tablas estadísticas en libros de texto chilenos de $1^{\circ}$ y $2^{\circ}$ año de Educación Primaria. Revista Paranaense de Educação Matemática, Campo Mourão, v. 4, n. 7, p. 10-39, 2015.

ESPINOZA, N. Tablas y gráficos de barras a través del ciclo del pensamiento estadístico. Un estudio con alumnos de primer grado de Educación Primaria. 2015. 92f. Tesis (Magíster en Enseñanza de las Matemáticas) - Escuela de Posgrado, Pontificia Universidad Católica del Perú, Lima, 2015.

ESTRELLA, S.; OLFOS, R. Transnumeración de los datos: el caso de las tablas de frecuencia. In: SCOTT, P.; RUÍZ, A. (Ed.). Educación Matemática en las Américas: 2015. Estadística y Probabilidad. Santo Domingo: Comité Interamericano de Educación, 2015. Matemática, v. 8. p. 220225.

EUDAVE, D. Niveles de comprensión de información y gráficas estadísticas en estudiantes de centros de educación básica para jóvenes y adultos de México. Educación Matemática, México, v. 21, n. 2, p. 5-37, 2009.

GABUCIO, F. et al. Niveles de comprensión de las tablas en alumnos de primaria y secundaria. Cultura y Educación, Barcelona, v. 22, n. 2, p. 183 - 197, 2010.

GAL, I. Adult's statistical literacy: Meaning, components, responsibilities. International Statistical Review, The Netherlands, v. 70, n. 1, p. 1-25, 2002.

GUIMARÃES, G. et al. Análise das atividades sobre representações gráficas nos livros didáticos de matemática. In: SIMPÓSIO INTERNACIONAL DE PESQUISA EM EDUCAÇÃO MATEMÁTICA. 2., 2008. Recife. Anais... Recife: UFRPE, 2008. p. 1-12.

HERNÁNDEZ, R.; FERNÁNDEZ, C.; BAPTISTA, P. Metodología de la Investigación. México: McGraw Hill, 2010.

HIRSCH, J. La comprensión lectora requiere conocimiento de vocabulario y del mundo hallazgos científicos sobre el bajón de cuarto grado y el estancamiento en los puntajes nacionales de comprensión. Estudios Públicos, Santiago, n. 108, p. 230-252, 2007.

MARTON, F.; HOUNSELL, D.J.; ENTWISTLE, N. The experience of learning. Edimburgo: Scottish Academic Press, 1984. 
MINISTÉRIO DA EDUCAÇÃO - MEC. Parâmetros Curriculares Nacionais: Matemática, Ensino de 1a à 4a série. Brasília: Secretaria de Educação Fundamental, 1997.

MINISTERIO DE EDUCACIÓN, CULTURA Y DEPORTE - MECD. Real Decreto 126/2014, de 28 de febrero, por el que se establece el currículo básico de la Educación Primaria. Madrid: Boletín Oficial del Estado, $n^{\circ}$ 52, 2014.

MINISTERIO DE EDUCACIÓN - MINEDUC. Matemática educación básica. Bases curriculares. Santiago: Unidad de Currículum y Evaluación, 2012.

NATIONAL COUNCIL OF TEACHERS OF MATHEMATICS - NCTM. Principles and standards for school mathematics. Reston: Autor, 2000.

OCHOA, E. El ciclo de investigación del pensamiento estadístico relacionado con tablas de doble entrada. Un estudio con alumnos del segundo grado de Educación Primaria. 2015. 113f. Tesis (Magíster en Enseñanza de las Matemáticas) - Escuela de Posgrado, Pontificia Universidad Católica del Perú, Lima, 2015.

PEREIRA, E. L.; CONTI, K. C. Interpretando tabelas e construindo gráficos com alunos do $3^{\circ}$ ano do Ensino Fundamental. In: TOMMASIELLO, M. et al. (Ed.). Didática e Práticas de Ensino na Realidade Escolar Contemporânea: constatações, análises e proposições. Araraquara: Junqueira \& Marin, 2012. p. 5294-5302.

SEPÚLVEDA, A. El aprendizaje de la Biología desde la taxonomía SOLO: Niveles SOLO en estudiantes de enseñanza media. 2004 340f. Tesis (Doctorado en Educación) - Facultad de Educación, Universidad Academia de Humanismo Cristiano, Santiago, 2004.

SEPÚLVEDA, A.; DELGADO, H. Cuánto aprenden los estudiantes de colegios públicos y privados. Revista de Estudios y Experiencias en Educación, Concepción, v. 6, n. 12, p. 73-90, 2007.

STONE, M. La enseñanza para la comprensión. Vinculación entre la investigación y la práctica. Buenos Aires: Paidós, 1999.

WATSON, J. Assessing statistical literacy through the use of media surveys. In: GAL, I; GARFIELD, J. B. (Ed.). The assessment challenge in statistics education. Amsterdam: IOS Press, 1997. p. 107121. 
ANEXO 1:

\section{CUESTIONARIO SOBRE TABLAS ESTADÍSTICAS PARA ESTUDIANTES DE EDUCACIÓN BASICA}

\section{ACTIVIDAD EVALUATIVA 1. Lee la siguiente situación y responde las preguntas.}

A un grupo de estudiantes de tercero básico se le aplicó una encuesta y las respuestas a una de las preguntas se organizaron en la siguiente tabla.

Según la tabla:

a) ¿Cuál es el título de la tabla?

b) ¿Qué asignatura obtuvo la mayor votación?

c) ¿Cuál es la cantidad total de estudiantes que respondieron a la pregunta? Escribe el proceso seguido.

d) Escribe la pregunta que tú crees que fue

\begin{tabular}{|c|c|c|}
\hline \multicolumn{3}{|c|}{ Asignaturas preferidas } \\
\hline Asignatura & Conteo & Cantidad de respuestas \\
\hline Lenguaje & \|\| & 4 \\
\hline Matemática & \|\|\|\|\|\| & 9 \\
\hline Educación Física & \|\|\|\| & 7 \\
\hline Ciencias naturales & \|\|$\|$ & 6 \\
\hline
\end{tabular}
planteada a los estudiantes para obtener la información de la tabla.

\section{ACTIVIDAD EVALUATIVA 2. Lee la siguiente situación y desarrolla las actividades.}

A cada uno de los estudiantes de un curso se le preguntó por su color favorito. Las respuestas de cada uno de ellos fueron organizadas en la siguiente tabla:

a) ¿Qué color fue indicado como preferido por un total de 7 estudiantes?

b) ¿Cuál es la diferencia entre el color de mayor y menor preferencia?

c) Si dos personas más participan de la votación y deciden cambiar el color de mayor votación, por qué color debieran votar?

d) María afirma que "si se hace la misma pregunta todos los estudiantes de la escuela los resultados serán similares". Justifica si estás de acuerdo, o no, con esta afirmación.

\begin{tabular}{|l|c|}
\hline \multicolumn{2}{|c|}{ Color preferido } \\
\hline Color & Cantidad \\
\hline Rojo & 8 \\
\hline Azul & 7 \\
\hline Amarillo & 4 \\
\hline Verde & 5 \\
\hline
\end{tabular}

\section{ACTIVIDAD EVALUATIVA 3. Lee la siguiente situación y desarrolla las actividades.}

A los estudiantes se les preguntó sobre su fruta preferida. Los resultados se organizaron en la siguiente tabla.

a) ¿Cuántos estudiantes prefieren la piña?

b) ¿Cuántos estudiantes en total prefieren las frutillas y las naranjas?

c) Invente un problema que se puede resolver con la información presente en la tabla

d) Construye un gráfico estadístico con la información mostrada en la tabla.

\begin{tabular}{|c|c|}
\hline \multicolumn{2}{|c|}{ Frutas favoritas } \\
\hline Fruta & Cantidad \\
\hline Frutilla & 6 \\
\hline Piña & 10 \\
\hline Durazno & 6 \\
\hline Naranja & 8 \\
\hline
\end{tabular}

\section{ACTIVIDAD EVALUATIVA 4. Lee la siguiente situación y responde las preguntas.}

En la siguiente tabla se muestra el nivel (en porcentaje: \%) de aprobación y reprobación del desempeño de la directora del jardín infantil "Caracolito" durante los últimos nueve meses de su gestión.

\begin{tabular}{|l|c|c|c|c|c|c|c|c|c|}
\hline \multicolumn{10}{|c|}{ Opinión de las personas por mes } \\
\hline \multicolumn{1}{|c|}{ Criterio } & Mes 1 & Mes 2 & Mes 3 & Mes 4 & Mes 5 & Mes 6 & Mes 7 & Mes 8 & Mes 9 \\
\hline Aprobación del desempeño & $54 \%$ & $53 \%$ & $56 \%$ & $58 \%$ & $54 \%$ & $49 \%$ & $47 \%$ & $45 \%$ & $42 \%$ \\
\hline Desaprobación del desempeño & $20 \%$ & $32 \%$ & $31 \%$ & $32 \%$ & $36 \%$ & $41 \%$ & $45 \%$ & $47 \%$ & $52 \%$ \\
\hline
\end{tabular}

De acuerdo a la información mostrada en ella, responda las siguientes preguntas.

a) ¿Qué porcentaje de desaprobación alcanzó en el mes 5?

b) ¿Cuánto ha variado la aprobación durante los nueve meses indicados en la tabla?

c) ¿Cuál será el porcentaje de aprobación que tendrá la directora del Jardín en el décimo mes? Explica.

d) ¿Qué actividades educativas podría hacer la directora para aumentar su nivel de aprobación? 